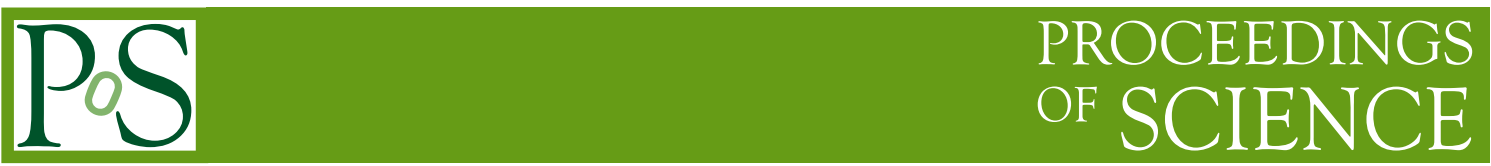

\title{
CP violation with the ATLAS and CMS experiments
}

\author{
Adam Edward Barton, On behalf of the ATLAS Collaboration ${ }^{a, *}$ \\ ${ }^{a}$ Lancaster University, \\ Lancaster, UK \\ E-mail: adam.edward.barton@cern.ch
}

In the Standard Model of particle physics, $\mathrm{CP}$ violation arises due to a single complex phase in the Cabibbo-Kobayashi-Maskawa (CKM) quark mixing matrix. Testing the validity of the CKM mechanism as a source of $\mathrm{CP}$ violation is one of the major experimental challenges in particle physics today. Precise measurement of the CKM parameters therefore constrains the Standard Model, and may reveal effects beyond it. Measurement of the time-dependent decay rates of $B_{s}^{0} \rightarrow J / \psi \phi$ provides a theoretically clean method for extracting the CP-violating weak mixing phase $\phi_{s}$. The Standard Model predicts $\phi_{s}$ to be very small and it is very well constrained, while in many new physics models large $\phi_{s}$ values are expected. Small deviations in a measurement of $\phi_{s}$ would be hints for the existence of the new particles. The most recent results on the CP-violating mixing phase $\phi_{s}$ and several other parameters describing the $B_{s}^{0}$ meson system are presented from ATLAS and CMS, using $\sqrt{s}=13 \mathrm{TeV}$ proton-proton collision data from the LHC, are presented.

The Ninth Annual Conference on Large Hadron Collider Physics - LHCP2021

7-12 June 2021

Online

${ }^{*}$ Speaker 


\section{Introduction}

ATLAS [1] and CMS [2] are general purpose detectors that measure heavy-flavour properties using their inner detectors, muon spectrometers and electromagnetic calorimeters. Measuring the properties of heavy-flavour particles has been part of the B physics program of the ATLAS and CMS experiments since the start of the proton-proton $(p p)$ collisions at the LHC in 2010. The ATLAS analysis presented here [3] introduces a measurement of the $B_{s} \rightarrow J / \psi \phi$ decay parameters using $80.5 \mathrm{fb}^{-1}$ of LHC $p p$ data collected by the ATLAS detector during $2015-2017$ at a centre-of-mass energy, $\sqrt{s}=13$. The CMS analysis presented here [4] shows a measurement of the same decay using $96.4 \mathrm{fb}^{-1}$ of $p p$ data collected by the CMS detector during $2017-2018$ at a centre-of-mass energy, $\sqrt{s}=13$.

In the presence of New Physics (NP) phenomena, sources of CP violation in $b$-hadron decays can arise in addition to those predicted by the Standard Model (SM) [5]. In the $B_{s} \rightarrow J / \psi \phi$ decay, $\mathrm{CP}$ violation occurs due to interference between a direct decay and a decay with $B_{s}-\bar{B}_{s}^{0}$ mixing. The oscillation frequency of $B_{s}$ meson mixing is characterised by the mass difference $\Delta m_{s}$ of the heavy $\left(B_{\mathrm{H}}\right)$ and light $\left(B_{\mathrm{L}}\right)$ mass eigenstates. The $\mathrm{CP}$ violating phase $\phi_{s}$ is defined as the weak phase difference between the $B_{s}-\bar{B}_{s}^{0}$ mixing amplitude and the $b \rightarrow c \bar{c} s$ decay amplitude. In the SM the phase $\phi_{s}$ is small and is related to Cabibbo-Kobayashi-Maskawa (CKM) quark mixing matrix elements via the relation $\phi_{s} \simeq-2 \beta_{s}$, with $\beta_{s}=\arg \left[-\left(V_{t s} V_{t b}^{*}\right) /\left(V_{c s} V_{c b}^{*}\right)\right]$; assuming no NP contributions to $B_{s}$ mixing and decays, a value of $-2 \beta_{s}=-0.0363_{-0.0015}^{+0.0016} \mathrm{rad}$ can be predicted by combining beauty and kaon physics observables [6]. While large NP enhancements of the mixing amplitude have been excluded by the precise measurement of the oscillation frequency [7], the NP couplings involved in the mixing may still increase the size of the observed CP violation by enhancing the mixing phase $\phi_{s}$ with respect to the SM value.

Other physical quantities involved in $B_{s}-\bar{B}_{s}^{0}$ mixing are the decay width $\left(\Gamma_{s}=\left(\Gamma_{\mathrm{L}}+\Gamma_{\mathrm{H}}\right) / 2\right)$ and the width difference $\left(\Delta \Gamma_{s}=\Gamma_{\mathrm{L}}-\Gamma_{\mathrm{H}}\right)$, where $\Gamma_{\mathrm{L}}$ and $\Gamma_{\mathrm{H}}$ are the decay widths of the light and heavy mass eigenstates, respectively. A potential NP enhancement of $\phi_{s}$ would also decrease the size of $\Delta \Gamma_{s}$, though it is not expected to be affected as significantly as $\phi_{s}$ [8].

\section{Flavour Tagging}

Opposite side tagging is used to infer the initial flavour of the B meson by producing a probability of whether it is a particle or anti-particle. ATLAS uses 4 methods selecting the one with the most power available in each event, "Tight Muons", Electron, "Low- $p_{T}$ Muon" and Jet. The effeciency, dilution and power of these can be seen in Table 1 (left) with the CMS muon tagging details separated down by data sample in Table 1 (right).

\section{Maximum likelihood fit}

An unbinned maximum-likelihood fit is performed on the selected events to extract the parameter values of the $B_{s} \rightarrow J / \psi\left(\mu^{+} \mu^{-}\right) \phi\left(K^{+} K^{-}\right)$decay. The fit uses information about the reconstructed mass $m$, the measured proper decay time $t$, the measured proper decay time uncertainty $\sigma_{t}$, the tagging probability, and the transversity angles $\Omega$ of each $B_{s} \rightarrow J / \psi \phi$ decay candidate. The 


\begin{tabular}{l|c|c}
\hline Tag method & Signal efficiency [\%] & Background efficiency [\%] \\
\hline \hline Tight muon & $4.06 \pm 0.06$ & $3.21 \pm 0.01$ \\
Electron & $1.86 \pm 0.04$ & $1.48 \pm 0.01$ \\
Low- $p_{\mathrm{T}}$ muon & $2.95 \pm 0.05$ & $2.70 \pm 0.01$ \\
Jet & $12.1 \pm 0.1$ & $9.41 \pm 0.02$ \\
Untagged & $79.1 \pm 0.3$ & $83.20 \pm 0.05$
\end{tabular}

\begin{tabular}{cccc} 
Data sample & $\varepsilon_{\text {tag }}(\%)$ & $\omega_{\text {tag }}(\%)$ & $P_{\text {tag }}(\%)$ \\
\hline 2017 & $45.7 \pm 0.1$ & $27.1 \pm 0.1$ & $9.6 \pm 0.1$ \\
2018 & $50.9 \pm 0.1$ & $27.3 \pm 0.1$ & $10.5 \pm 0.1$
\end{tabular}

Table 1: A Table showing the tagging methods used in the ATLAS analysis [3] (left). A Table showing the tagging power of the samples used in the CMS analysis [4] (right).

measured proper decay time uncertainty $\sigma_{t}$ is calculated from the covariance matrix associated with the vertex fit of each candidate event. The transversity angles $\Omega=\left(\theta_{\mathrm{T}}, \psi_{\mathrm{T}}, \phi_{\mathrm{T}}\right)$ are defined in Ref. [3]. In both analysis the likelihood was taken as independent of the invariant mass of $K^{+} K^{-}$ pair.

\section{Results}

The full simultaneous unbinned maximum-likelihood fit contains nine physical parameters: $\Delta \Gamma_{s}, \phi_{s}, \Gamma_{s},\left|A_{0}(0)\right|^{2},\left|A_{\|}(0)\right|^{2}, \delta_{||}, \delta_{\perp},\left|A_{S}(0)\right|^{2}$ and $\delta_{S}$. The other parameters in the likelihood function are the $B_{s}$ signal fraction $f_{s}$, parameters describing the $J / \psi \phi$ mass distribution, parameters describing the decay time plus angular distributions of background events, parameters used to describe the estimated decay time uncertainty distributions for signal and background events, and scale factors between the estimated decay time uncertainties and their true uncertainties.

The function finds two minima in the ATLAS data sample, these minima show identical values for the primary physical quantities $\phi_{s}, \Delta \Gamma_{s}, \Gamma_{s}$ and helicity amplitudes, while different values for strong phases $\delta_{\|}$and $\delta_{\perp}$, both results are presented in Table 2 (left). The CMS results are also presented in Table 2 (right).

\begin{tabular}{|c|c|c|c|c|c|c|c|}
\hline Parameter & Value & $\begin{array}{c}\text { Statistical } \\
\text { uncertainty }\end{array}$ & $\begin{array}{l}\text { Systematic } \\
\text { uncertainty }\end{array}$ & \multirow[b]{4}{*}{ Parameter } & \multirow[b]{4}{*}{ Fit value } & \multirow[b]{4}{*}{ Stat. uncer. } & \multirow{9}{*}{$\begin{array}{l}\text { Syst. uncer. } \\
\pm 10 \\
\pm 0.007 \\
\pm 0.03 \\
\pm 0.008 \\
\pm 0.0024 \\
\pm 0.0048\end{array}$} \\
\hline$\phi_{s}[\mathrm{rad}]$ & 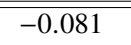 & 0.041 & 0.022 & & & & \\
\hline$\Delta \Gamma_{s}\left[\mathrm{ps}^{-1}\right]$ & 0.0607 & 0.0047 & 0.0043 & & & & \\
\hline$\Gamma_{s}\left[\mathrm{ps}^{-1}\right]$ & 0.6687 & 0.0015 & 0.0022 & & & & \\
\hline$\left|A_{\|}(0)\right|^{2}$ & 0.2213 & 0.0019 & 0.0023 & $\phi_{\mathrm{s}}[\mathrm{mrad}]$ & -11 & \pm 50 & \\
\hline$\left|A_{0}(0)\right|^{2}$ & 0.5131 & 0.0013 & 0.0038 & $\Delta \Gamma_{\mathrm{s}}\left[\mathrm{ps}^{-1}\right]$ & 0.114 & \pm 0.014 & \\
\hline$\left|A_{S}(0)\right|^{2}$ & 0.0321 & 0.0033 & 0.0046 & $\Delta m_{\mathrm{s}}\left[\hbar \mathrm{ps}^{-1}\right]$ & 17.51 & $\begin{array}{l}+0.10 \\
{ }_{-0.09}\end{array}$ & \\
\hline \multirow{2}{*}{$\delta_{\perp}-\delta_{S}[\mathrm{rad}]$} & -0.25 & 0.05 & 0.04 & & 0.972 & \pm 0.026 & \\
\hline & \multicolumn{3}{|l|}{ Solution (a) } & $\begin{array}{l}\Gamma_{\mathrm{s}}\left[\mathrm{ps}^{-}\right. \\
\left.A_{\mathrm{N}}\right|^{2}\end{array}$ & $\begin{array}{l}0.6531 \\
0.5350\end{array}$ & $\begin{array}{l} \pm 0.0042 \\
+0.0047\end{array}$ & \\
\hline$\delta_{\perp}[\mathrm{rad}]$ & 3.12 & 0.11 & 0.06 & $\begin{array}{l}\left|A_{0}\right|^{2} \\
\left|A_{\perp}\right|^{2}\end{array}$ & $\begin{array}{l}0.3350 \\
0.2337\end{array}$ & $\begin{array}{l} \pm 0.004 / \\
\pm 0.0063\end{array}$ & $\begin{array}{l} \pm 0.0048 \\
\pm 0.0044\end{array}$ \\
\hline \multirow[t]{2}{*}{$\delta_{\|}[\mathrm{rad}]$} & 3.35 & 0.05 & 0.09 & $\left|A_{\mathrm{S}}\right|^{2}$ & 0.022 & +0.008 & \pm 0.016 \\
\hline & \multicolumn{3}{|l|}{ Solution (b) } & $\delta_{\|}[\mathrm{rad}]$ & 3.18 & \pm 0.12 & \pm 0.03 \\
\hline$\delta_{\perp}[\mathrm{rad}]$ & 2.91 & 0.11 & 0.06 & $\delta_{\perp}[\mathrm{rad}]$ & 2.77 & \pm 0.16 & \pm 0.04 \\
\hline$\delta_{\|}[\mathrm{rad}]$ & 2.94 & 0.05 & 0.09 & $\delta_{\mathrm{S} \perp}[\mathrm{rad}]$ & 0.221 & $\begin{array}{l}+0.083 \\
-0.070\end{array}$ & \pm 0.048 \\
\hline
\end{tabular}

Table 2: A Table showing the physical parameters measured in the ATLAS dataset [3] (left). A Table showing the physical parameters measured in the CMS dataset [4]. (Right)

Both experiments performed a statistical combination of their new results with those obtained in Run1 $[9,10]$ using the BLUE method. This method uses the measured values and uncertainties 
of the parameters as well as the correlations between them. These can been seen as contours in Fig. 1 .
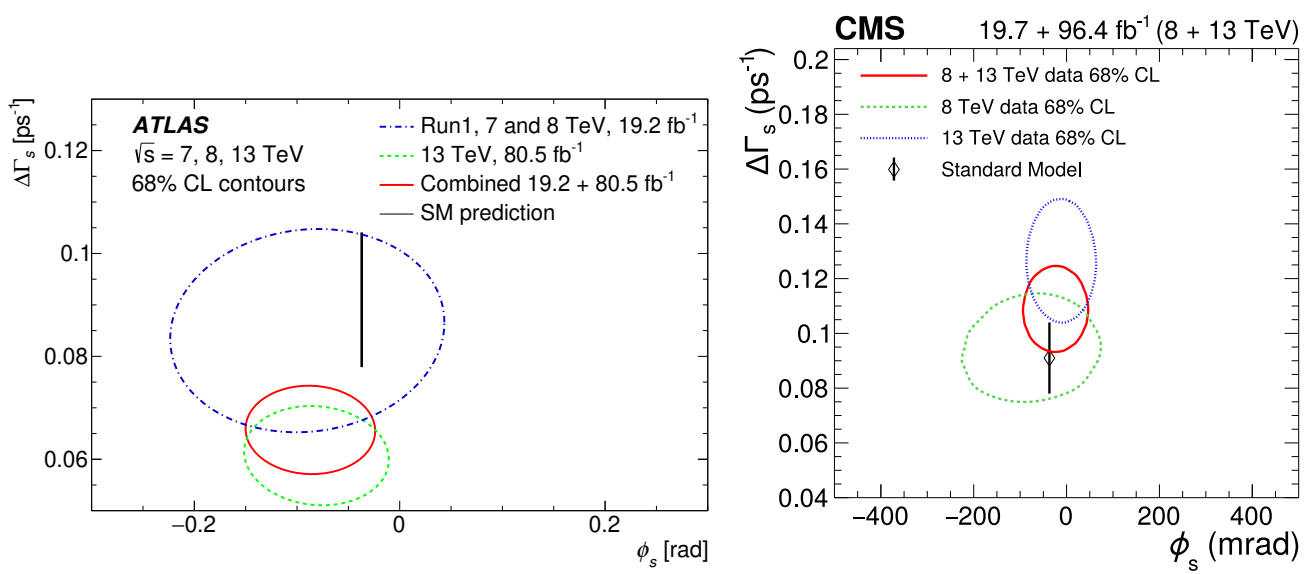

Figure 1: A figure showing the physical parameters when combined with Run 1 using the ATLAS data (left) and the CMS data (right)

The Heavy Flavour Averaging Group has contour plots showing a comparision of results from different analyses [11], these can be seen in Fig. 2. Results on $\phi_{s}$ are compatible between ATLAS and CMS and with SM, while there are some tensions in $\Delta \Gamma$ results.

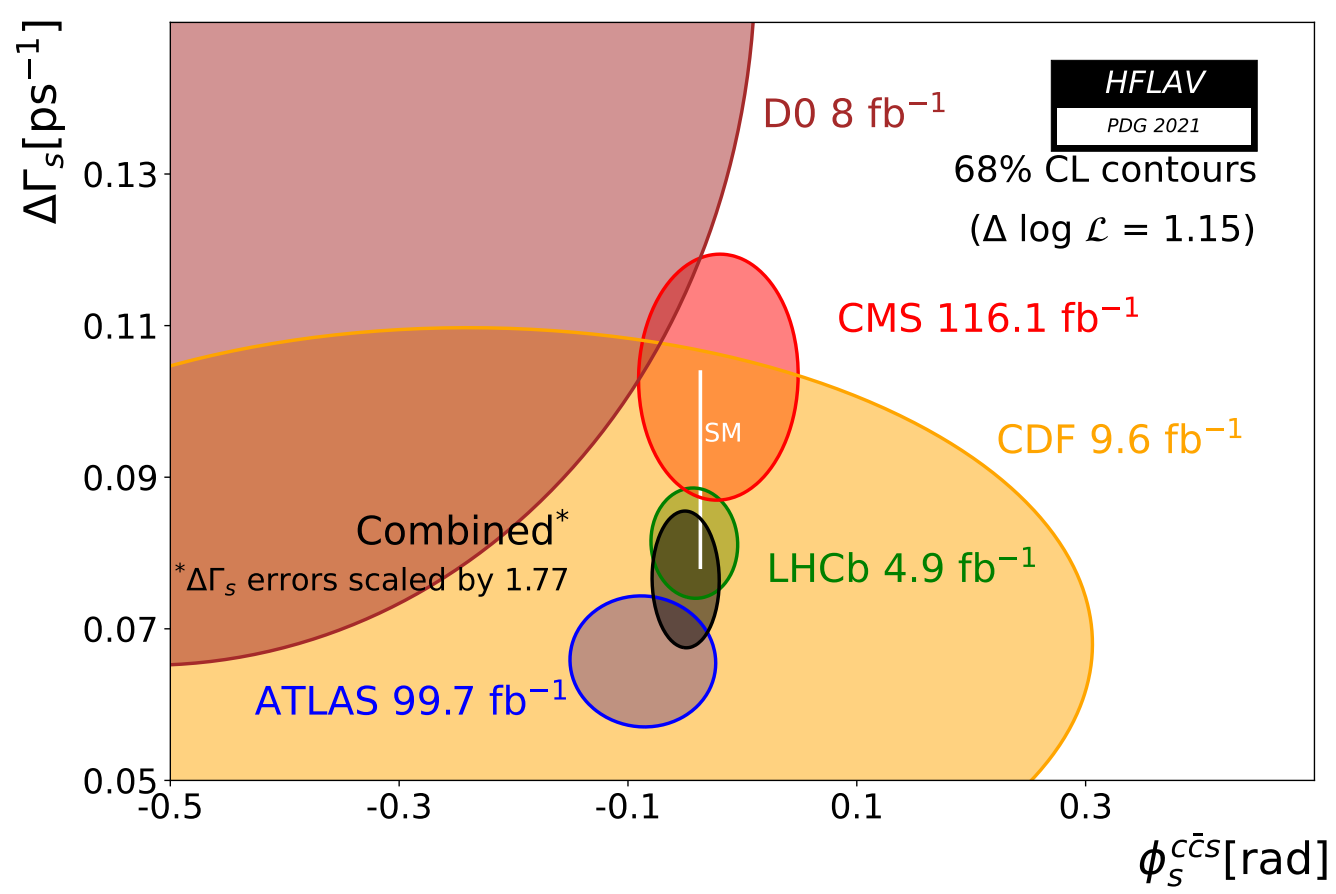

Figure 2: The FLAV plots show the contours of ATLAS, CMS, CDF, D0 and LHCb [11]. 


\section{References}

[1] ATLAS Collaboration, The ATLAS Experiment at the CERN Large Hadron Collider, 2008 JINST 3S08003

[2] CMS Collaboration, The CMS experiment at the CERN LHC. The Compact Muon Solenoid experiment, 2008 JINST S08004

[3] ATLAS Collaboration, Measurement of the CP-violating phase $\phi_{s}$ in $B_{S} \rightarrow J / \psi \phi$ decays in ATLAS at $13 \mathrm{TeV}$, Eur. Phys. J. C 81 (2021) 342

[4] CMS Collaboration, Measurement of the CP-violating phase $\phi_{s}$ in the $B_{s} J / \psi \phi(1020) \rightarrow$ $\mu^{+} \mu^{-} K^{+} K^{-}$channel in proton-proton collisions at $\sqrt{s}=13 \mathrm{TeV}$, Phys. Lett. B 816 (2021) 136188

[5] F. J. Botella, G. C. Branco and M. Nebot, $C P$ violation and limits on New Physics including recent $B_{s}$ measurements, Nuclear Physics B 768 (2007) 1.

[6] J. Charles et al., Predictions of selected flavour observables within the standard model, Phys. Rev. D 84 (2011) 033005

[7] LHCb Collaboration, Precision measurement of the $B_{s}^{0}-\bar{B}_{s}^{0}$ oscillation frequency with the decay $B_{s}^{0} \rightarrow D_{s}^{-} \pi^{+}$, New Journal of Physics 15 (2013) 053021, arXiv: 1304.4741 [hep-ex].

[8] A. Lenz, and U. Nierste Theoretical update of $B_{s}-\bar{B}_{s}$ mixing, JHEP 06 (2007) 072

[9] ATLAS Collaboration, Measurement of the CP-violating phase $\phi_{s}$ in $B_{S} \rightarrow J / \psi \phi$ decays in ATLAS at $8 \mathrm{TeV}$, JHEP 08 (2016) 147

[10] CMS Collaboration, Measurement of the CP-violating phase $\phi_{s}$ in the $B_{s} J / \psi \phi(1020) \rightarrow$ $\mu^{+} \mu^{-} K^{+} K^{-}$channel in proton-proton collisions at $\sqrt{s}=8 \mathrm{TeV}$, Phys. Lett. B 757 (2016) 97

[11] Y. Amhis et al. (Heavy Flavour Averaging Group), "Averages of b-hadron, c-hadron, and $\tau$-lepton properties as of summer 2018" 